\title{
Infective endocarditis affecting the eustachian valve
}

\author{
A D EDWARDS, M A VICKERS, C J MORGAN \\ From the Cardiac Department and Intensive Care Unit, Brompton Hospital, London
}

SUMMARY A previously fit 44 year old man presented with acute staphylococcal pneumonia. Despite appropriate antibiotics he showed signs of continuing sepsis and eventually died. At necropsy endocarditis of the eustachian valve was found.

In the fetus the eustachian valve (valve of the inferior vena cava) directs blood flow preferentially from the inferior vena cava through the foramen ovale into the left atrium. In the adult it is redundant and usually rudimentary ${ }^{1}$ although sometimes it is large enough to form a septum dividing the right atrium and creating cor triatriatum dexter. ${ }^{2}$

Infective endocarditis is known to complicate congenital heart anomalies but we are not aware of a previous description of endocarditis affecting the eustachian valve.

\section{Case report}

A 44 year old fireman who had no history of previous illness or intravenous drug abuse was admitted with right upper lobe pneumonia. Sputum repeatedly grew Staphylococcus aureus and he was treated with appropriate antibiotics. Over the next month the pneumonia progressed to a lung abscess, which discharged leaving a thin walled pneumatocele. During this time he had persistent hyperglycaemia and hyponatraemia and several episodes of septicaemia. Various organisms were cultured from blood and sputum and he was treated appropriately. His clinical condition did not improve despite discharge of pus and the continued use of antibiotics. An echocardiogram was performed which showed no evidence of endocarditis on the mitral, aortic, tricuspid, or pulmonary valves. A valve of the inferior vena cava was not specifically sought. Disseminated intravascular coagulation and acute renal failure devel-

Requests for reprints to Dr C J Morgan, Brompton Hospital, Fulham Road, London SW36HP.

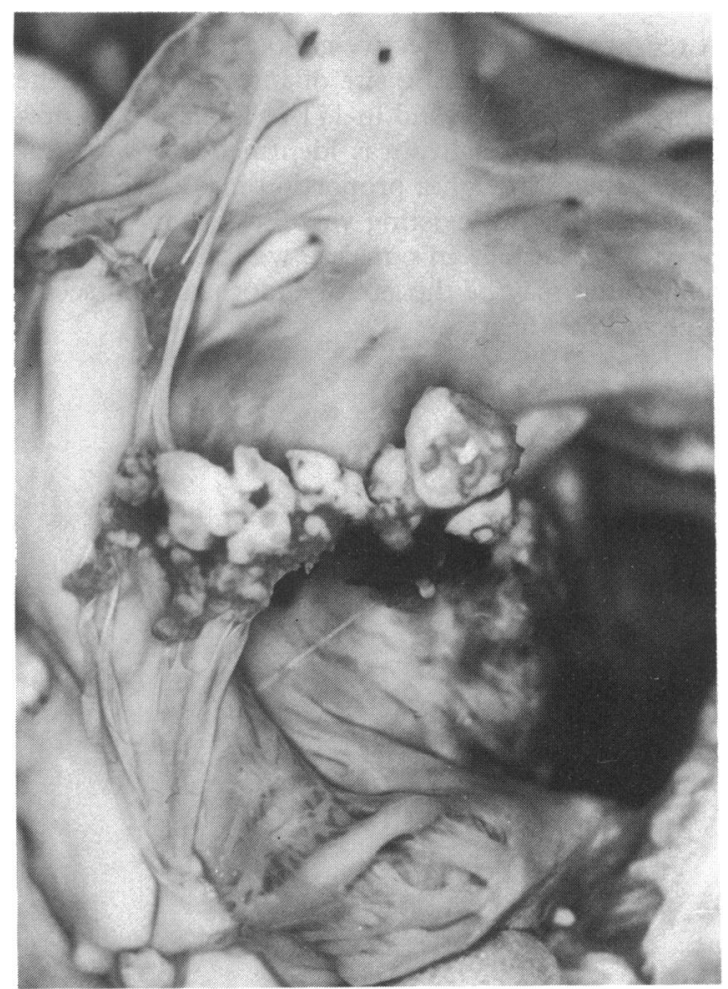

The eustachian valve, covered with large vegetations, crosses the right atrium with its chordae tendineae extending above and below. The dark area, bottom right, is the orifice of the tricuspid valve; the oval fossa is evident above the eustachian valve. 
oped and he continued to deteriorate. He died seven weeks after admission.

Necropsy confirmed the pneumatocele and there was patchy interstitial fibrosis with alveolar type II cell hyperplasia throughout the lungs that was suggestive of a recent viral infection. In the right atrium there was a well developed eustachian valve and hanging from it were large vegetations (figure). There were also small sessile vegetations on the mitral valve cusps. The eustachian valve vegetations consisted of fibrin containing collections of neutrophils but no colonies of bacteria: we considered that they were likely to represent bacterial endocarditis sterilised by the antibiotics that had been administered throughout the patient's illness. The mitral valve vegetations consisted only of fibrin and appeared to be marantic lesions. The heart was otherwise normal. Diffuse intravascular coagulation, pituitary necrosis, renal cortical necrosis, and chronic pancreatitis were noted.

\section{Discussion}

A recent survey of infective endocarditis found that in 199 of 544 cases the site of infection within the heart was uncertain and in 171 of 541 cases no predisposing abnormality was identified. ${ }^{3}$ It was suggested that although a proportion may have had an unrecognised pre-existing valve lesion, in most the heart was normal. In our case we found an abnormality that may be the site of endocarditis in seemingly normal hearts.
Echocardiography is a valuable investigation in the patient with suspected endocarditis and when it is performed the major heart valves are always carefully examined. Right atrial structures such as the eustachian valve and the Chiari network can be visualised well by cross sectional endocardiography. ${ }^{24}$ The present case suggests that when bacterial endocarditis is suspected and the four major valves are examined and appear normal, a valve of the inferior vena cava may be present. If a eustachian valve is visualised echocardiographically it should be considered as a rare site for vegetations.

We thank Professor R H Anderson, Professor B Corrin, and Dr Y Ho for their advice.

\section{References}

1 Warwick R, Williams PL. Gray's anatomy. 35th ed. London: Longman, 1973:601 and 613.

2 Fukaya T, Tomita Y, Baba K, et al. M-mode and realtime two-dimensional endocardiographic findings of the persistent sinus venosus valve. $f$ Cardiogr 1981;11:277-88.

3 Bayliss R, Clarke C, Oakley CM, Somerville W, Whitfield AGW, Young SEJ. The microbiology and pathogenesis of infective endocarditis. Br Heart $\mathcal{F}$ 1983;50:513-9.

4 Okamoto M, Beppu S, Nagata S, et al. Echocardiographic features of the Eustachian valve and its clinical significance. $\mathcal{F}$ Cardiogr 1981;11:271-6. 